\title{
Association Between Multiparametric Magnetic Resonance Imaging of the Prostate and Oncological Outcomes after Primary Treatment for Prostate Cancer: a Systematic Review and Meta-analysis
}

\author{
Armando Stabile ${ }^{1}$, Elio Mazzone ${ }^{1}$, Giuseppe O Cirulli ${ }^{1}$, Francesco De Cobelli ${ }^{2}$, Jeremy \\ Grummet $^{3}$, Harriet C Thoeny ${ }^{4}$, Mark Emberton ${ }^{5}$, Morgan Pokorny ${ }^{6}$, Peter A Pinto ${ }^{7}$, Samir \\ S Taneja ${ }^{8}$
}

${ }^{1}$ Department of Urology and Division of Experimental Oncology, URI, Urological Research Institute, Vita-Salute San Raffaele University, IRCCS San Raffaele Scientific Institute, Milan, Italy

${ }^{2}$ Department of Radiology, Vita-Salute San Raffaele University, IRCCS San Raffaele Scientific Institute, Milan, Italy

${ }^{3}$ Department of Surgery, Central Clinical School, Monash University, Melbourne, VIC, Australia

${ }^{4}$ Department of Radiology, Hôpital Cantonal de Fribourg HFR, University of Fribourg, Fribourg, Switzerland

${ }^{5}$ UCL Division of Surgery \& Interventional Science, University College London, London, UK; Department of Urology, University College London Hospitals NHS Foundation Trust, London, UK

${ }^{6}$ Department of Urology, Auckland City Hospital, Auckland, New Zealand

${ }^{7}$ Urologic Oncology Branch, National Cancer Institute, National Institutes of Health, Bethesda, MD, USA

${ }^{8}$ Department of Urologic Oncology, NYU Langone Medical Center, New York, New York 


\section{Corresponding author:}

Armando Stabile, MD

Department of Urology and Division of Experimental Oncology

Urological Research Institute, IRCCS San Raffaele Scientific Institute,

Via Olgettina 60, 20132 Milan, Italy

Tel. +39 02 26435663; Fax. +3902 26437298

E-mail: armando.stabile88@gmail.com

Word count (text): 3244

Word count (abstract): 250
Text pages: 12

References: 47
Tables: 1

Figures: 4

Keywords: prostate cancer; multiparametric magnetic resonance imaging; MRI; oncological outcomes; prognosis;

This research received no specific grant from any funding agency in the public, commercial, or notfor-profit sectors. The authors declare no conflicts of interest in preparing this article. 


\section{Abstract}

2 Context: the diagnostic accuracy of multiparametric magnetic resonance imaging

3 (mpMRI) for prostate cancer (PCa) diagnosis has been extensively explored. Little is

4 known about the prognostic value of mpMRI suspicion scores and other quantitative 5 mpMRI information.

6 Objective: we aimed to systematically review the current literature assessing the

7 relationship between pre-treatment mpMRI and oncological outcomes after primary

8 treatment for PCa in order to assess the role of mpMRI as a prognostic tool.

9 Evidence acquisition: a computerized bibliographic search of Medline/PubMed,

10 EMBASE, Scopus, and Cochrane Library's Central databases was performed for all studies

11 assessing the relationship between mpMRI and oncological outcomes after primary

12 treatment for PCa. The review protocol was published in the PROSPERO database

13 (CRD42020209899).

14 Evidence synthesis: a total of 6 studies were included. Reliable evidence is still limited in

15 this field. The PI-RADS score represented an independent predictor of biochemical

16 recurrence (BCR) after radical prostatectomy (RP) in the majority of the included studies.

17 The tumor volume at mpMRI was not significantly associated with BCR after RP for PCa.

18 Data on disease progression and PCa specific mortality is limited. Heterogeneity among

19 studies was substantial.

20 Conclusions: PI-RADS score appears, from this review, to provide information on the

21 future likelihood of cancer recurrence or progression at least in men receiving RP. We are

22 of the view that this information should be taken into account to identify men at higher risk

23 of unfavorable outcomes. 
24 Patient summary: higher PI-RADS score seems to be positively associated with

25 oncological failure and should be incorporated into future risk models.

26

27

28

29

30

31

32

33

34

35

36

37

38

39

40

41

42

43

44

45

46 


\section{1. Introduction}

48 The use of multiparametric magnetic resonance imaging (mpMRI) for prostate

49 cancer (PCa) diagnosis has significantly increased [1], and nowadays its use is strongly

50 suggested before prostate biopsy in any clinical setting [2]. Furthermore, mpMRI has also

51 been used to improve PCa staging accuracy [3,4]. Nonetheless, there is still a lack of

52 evidence supporting the value of mpMRI suspicion score in predicting oncological

53 outcomes after primary treatment for PCa. In fact, most of the existing predictive tools still

54 rely on clinical and pathological findings [5]. A recent meta-analysis showed that mpMRI

55 findings significantly affect PCa outcomes after primary treatment with radiotherapy [6].

56 Nonetheless, most of the studies included in this work were carried out before mpMRI

57 protocol standardization [7] and none of the findings that currently are considered to be

58 crucial in mpMRI reporting were tested. Indeed, mpMRI reporting assessment provides

59 standardized information based on the interpretation of the different sequences according

60 to a Likert score [8] or the more standardized Prostate Imaging Reporting and Data System

$61[7,9,10]$. These suspicion scores are in fact well-known predictors for the presence of PCa

62 among other predictors such as apparent diffusion coefficient (ADC) and tumor volume

63 that are usually provided by mpMRI $[11,12]$.

64 When considering treatment for $\mathrm{PCa}$, an accurate risk assessment is pivotal to

65 properly counsel patients, helping to identify those men at higher risk of adverse clinical

66 outcomes. Bearing this in mind, and given the crucial role that mpMRI has gained in the

67 diagnosis and staging of PCa, the inclusion of mpMRI metrics within the risk assessment

68 process of patients referred for PCa treatment needs to be addressed. 
This study aimed to systematically review the studies assessing the relationship

70 between measures of mpMRI suspicion and oncological outcomes after primary treatment

71 for PCa in order to explore whether the role of mpMRI as a prognostic tool in this setting

72 should be supported.

73

\section{2. Evidence acquisition}

\section{2.1. Objective}

76 We aimed to systematically review the current literature assessing the relationship

77 between pre-treatment mpMRI findings and oncological outcomes after primary treatment 78 for PCa.

\section{2.2. Search strategy}

81 Data collection was conducted in accordance with the Preferred Reporting Items

82 for Systematic Reviews and Meta-analyses (PRISMA) statement [13]. A computerized

83 bibliographic search of Medline/PubMed, EMBASE, Scopus, and Cochrane Library's

84 Central databases was performed from inception to September $1^{\text {st }}$, 2020. From this date

85 onwards, eventual relevant studies published were included in the analyses. The search

86 strategy used is summarized in Supplementary Methods - Appendix 1. The review protocol

87 was registered in the PROSPERO database (registration number: CRD42020209899).

88

\section{2.3. Inclusion and exclusion criteria}

90 As recommended by the PRISMA guidelines, we used the population, intervention,

91 comparator, and outcome (PICO) approach to define study eligibility [13]. Reports were 
92 considered relevant if they provided data regarding the relationship between mpMRI and

93 oncological outcomes after primary treatment for $\mathrm{PCa}$ (i.e. radical prostatectomy [RP],

94 external beam radiotherapy [EBRT], focal therapy, brachytherapy, others). More

95 specifically, only studies assessing the relationship between pre-treatment mpMRI

96 information related with the PCa index lesion (e.g. scoring system, index lesion tumor

97 volume [TV], ADC, etc.) and oncological outcomes were included. Oncological outcomes

98 were defined as one of the following: biochemical recurrence (BCR) after radical treatment

99 (RP and/or EBRT), disease progression (defined according to the criteria suggested by

100 Prostate Cancer Clinical Trials Working Group 2 [14]), metastatic failure (appearance of

101 metastasis after treatment), overall- or cancer specific-mortality.

102 The studies with the following characteristics were excluded: 1) studies that did not

103 use or provide any information regarding the mpMRI assessment/scoring system and did

104 not rely on standardized systems for reporting MRI (i.e. Prostate Imaging - Reporting And

105 Data System [PI-RADS] v1 or higher); 2) studies only assessing the prognostic value of

106 mpMRI staging; 3) studies with insufficient survival data needed for meta-analysis; 4)

107 when multiple studies relied on duplicated cohort, the one providing the most

108 comprehensive information was included; 5) case reports, editorials, letters, review

109 articles, and meeting abstracts.

110

\section{2.4. Systematic review process and data extraction}

112 Three authors (A.S., E.M. and G.C.) independently reviewed a total of 2,036

113 abstracts and selected 107 studies that were considered eligible for full-text evaluation and

114 eventual inclusion in the systematic review. Fig. 1 shows the PRISMA flowchart 
115 describing the selection process. Data were independently extracted from all included

116 studies by the same authors. Discrepancies between reviewers were resolved via consensus,

117 adjudicated by a third reviewer (E.M.).

119 2.5. Data analysis

120 The outcomes considered were BCR, disease progression, overall- and cancer

121 specific-mortality. A pooled analysis was performed when more than one study testing the

122 same mpMRI predictor and the same outcome was available. Alternatively, narrative

123 synthesis of the studies was performed. The effect size of mpMRI factors associated with

124 the outcomes was measured in terms of hazard ratios (HRs). The HRs and the

125 corresponding standard errors for each predictor were extracted from each study included

126 when available. Pooled analyses of the HRs for each mpMRI factor and the related outcome

127 were performed using the inverse variance technique for meta-analysis of HRs, specifically

128 the random-effects model according to DerSimonian and Laird $[15,16]$. In order to test

129 mpMRI factors as predictors of oncological outcomes after treatment for PCa, as suggested

130 by Cochrane Handbook for Meta-Analyses [15], only studies providing adjusted HRs were

131 included for pooled analyses, whereas studies providing unadjusted estimates were

132 excluded from the main analysis. Heterogeneity between studies was measured using the

$133 I^{2}$ statistics and the extent of the variation among the effects observed in different studies

134 (between-study variance) using $\tau^{2}$ from random-effect analyses [17]; $p$ values of $<0.05$

135 were considered to indicate statistical significance. Where the same study provided

136 different HRs for different cut-offs/populations, the same study was duplicated in the

137 analysis accordingly. All statistical analyses were performed using the RStudio graphical 
138 interface v.1.2.5033 for R software environment v.3.6.3. (packages included "metafor",

139 "meta" and "rms").

\section{2.6. Risk of bias assessment}

142 The risk of bias assessment of individual studies was assessed independently by the

143 same two authors using the Quality in Prognostic Studies tool [18] (Fig. 2).

\section{$145 \quad 3 . \quad$ Evidence synthesis}

146 Overall, 2,036 studies were initially screened after duplicates removal. If it was not

147 clear from the abstract whether the paper might contain relevant data, the full paper was

148 assessed. After full paper evaluation of the eligible studies $(n=107)$, six articles were

149 included in the final qualitative analysis according to the pre-specified inclusion criteria

150 (Fig. 1). Only studies reporting outcomes after radical prostatectomy (RP) were included

151 in the quantitative synthesis (i.e. meta-analysis). Given the significant between-studies

152 heterogeneity that may not be accounted for in a pooled meta-analysis, data on PI-RADS

153 score as a predictor of oncological outcomes was exclusively described in the qualitative

154 synthesis. Particularly, 5 studies were included in the narrative description on the effect of

155 PI-RADS score on BCR after RP and 2 studies in the meta-analysis testing the effect of

156 tumor volume at mpMRI on BCR after RP. Furthermore, one study tested the PI-RADS

157 score as a predictor for disease progression and one tested PI-RADS score as repdictor for

158 BCR in a cohort of men receiving radiotherapy.

159

160 3.1. Study and patient characteristics 
162 patients were included in the qualitative synthesis. The median age ranged between 59 [19]

163 and 69 [20] yr. A total of $1647(93 \%)$ and $123(7 \%)$ patients received radical prostatectomy

164 and radiotherapy, respectively. Among 1612 patients for whom clinical stage was

165 available, $1143(71 \%), 323(20 \%)$ and $146(9 \%)$ had clinical T1, T2 and T3 disease,

166 respectively. Among 1770 patients for whom Gleason score was available, 444 (25\%),

$1671108(63 \%)$ and $218(12 \%)$ had PCa with Gleason score 6,7 and $\geq 8$, respectively.

\section{3.2. Risk of bias within studies}

170 The overall risk of bias according to the Quality in Prognostic Studies tool [18] is

171 given in Fig.2. The overall methodological quality of the studies was moderate, with the 172 most significant issues concerning the study attrition and prognostic factors and 173 measurement domains mainly due to the retrospective nature of studies included and the 174 heterogeneity of the confounders considered within the adjusted analyses (Table 1 175 Supplementary Table 1). Supplementary Fig 1 shows the risk of bias for each study.

\section{3.3. Biochemical recurrence}

178 In total, 5 studies assessing mpMRI as a predictor of BCR after RP were included 179 in the qualitative synthesis. Specifically, 5 studies tested the mPMRI scoring system 180 [19,21-24], 2 studies tested the mpMRI TV [19,25], and one study measured the ADC of 181 the suspicious lesion as a predictor of BCR [20], respectively (Table 1). Two studies testing 182 the effect of mpMRI TV on outcomes were pooled in a quantitative meta-analysis. 
184 scoring system is shown in Table 1 and in Supplementary Figure 1. Three $[19,23,24]$ and 185 two studies [20,22] tested this predictor as a categoric and continuous variable, respectively 186 (Fig. 3). Among studies using PI-RADS score as categorical variable, Gandaglia et al. (804 187 patients) [19] recorded higher risk of BCR in patients with PI-RADS 5 vs 3 (HR 2.86, $18895 \%$ CI 1.14-7.20, $\mathrm{p}=0.04)$ but not in patients with PI-RADS 4 vs 3 (HR 1.48, 95\%CI $1890.60-3.64, \mathrm{p}=0.3)$ at mpMRI. Hattori et al. [23] (314 patients) confirmed higher risk of 190 BCR in patients with PI-RADS $\geq 3$ vs $<3$ (HR 6.19, 95\% CI 1.41-27.1, $\mathrm{p}=0.016)$ at mpMRI. 191 Similarly, Kim et al. [24] (166 patients) demonstrated higher probability of BCR in patients 192 with PI-RADS 5 vs $<5$ (HR 1.75, 95\% CI 1.05-2.93, p=0.033) and in patients with PI193 RADS $\geq 3$ vs $<3$ (HR 5.58, 95\%CI 1.34-23.2, p=0.018) at mpMRI, while no statistically 194 significant differences were recorded in patients with PI-RADS $\geq 4$ vs $<4$ at mpMRI (HR $1952.03,95 \%$ CI $0.95-4.36, \mathrm{p}=0.069$ ).

196 Among studies using PI-RADS score as continuous variable, Park et al. [22] relied 197 on 158 patients whose mpMRI were evaluated by two different radiologists. Here, PI198 RADS score resulted as independent predictor of BCR when mpMRI were evaluated by 199 the second reader (HR 2.78, 95\% CI 1.18-6.92, $\mathrm{p}=0.028)$, but such predictive effect was not 200 statistically significant when mpMRI were evaluated by the first reader (HR $2.46,95 \% \mathrm{CI}$ 201 0.95-6.41, p>0.05). Lastly, Zhang et al. [20] (205 patients) demonstrated that a higher PI202 RADS score was significantly associated with higher risk of BCR (HR 4.12, 95\%CI 1.07$20315.8, \mathrm{p}=0.039)$. 
205 studies assessed mpMRI TV after RP. Overall, mpMRI TV was not significantly associated 206 with BCR after RP for PCa (HR: 0.99; 95\% CI: 0.92-1.07; $\mathrm{p}=0.84$ )

207 Only one study [20] assessed ADC as a predictor of BCR providing an adjusted 208 HR, showing that the ADC value was negatively correlated with BCR-free survival (HR: $2091.747 ; 95 \%$ CI: $1.136-2.685 ; \mathrm{p}=0.011)$ meaning that a lower ADC value of the tumor before 210 treatment was associated with a worse outcome.

\section{3.4. Disease progression}

213 In total, one study assessing mpMRI as a predictor of disease progression after RP 214 was included [24]. In this study, Kim et al. relied on a population of 166 men receiving RP

215 for PCa. With a median follow-up of 9.1 years, the authors demonstrated that PI-RADS v2 216 was an independent predictor of disease progression (defined as local recurrence 217 [appearance of a new lesion in the prostatectomy bed], a new target lesion, lymph node 218 metastasis [ $\geq 2 \mathrm{~cm}$ in diameter], a bony lesion [appearance of two or more new lesions on 219 bone scan], and requirement for other therapies), after accounting for age, preoperative 220 PSA, performance status, clinical stage, biopsy Gleason score and number of positive 221 biopsy cores. More specifically, PI-RADS $<3$ vs. $\geq 3$, and PI-RADS $<4$ vs. $\geq 4$, and PI222 RADS $<5$ vs. 5 at multivariable Cox regression models were related with the rate of disease 223 progression with the following HRs: 3.99 (95\% CI: 0.96-16.59, p=0.047), 2.02 (95\% CI: $224 \quad 0.85-4.82, \mathrm{p}=0.113$ ), and 2.31 (95\% CI: 1.30-4.09, $\mathrm{p}=0.04)$, respectively.

\section{3.5. Biochemical recurrence in patients treated with radiotherapy}


Overall, one study tested the role of mpMRI in predicting BCR in patients treated

228 with EBRT [26]. In this study, 76 patients treated with EBRT were stratified according to

229 PI-RADS score $\leq 4$ or $>4$ at pre-EBRT mpMRI. At multivariable Cox model, patients with

230 PI-RADS $\geq 4$ treated with EBRT had higher rate of BCR (HR 5.37, 95\% CI: 1.55-25.3)

231 compared to patients with PI-RADS score $\leq 4$ at pre-EBRT mpMRI.

\section{4. Discussion}

234 The growing interest in the use of mpMRI for PCa diagnosis and the subsequent 235 amount of evidence supporting the accuracy of this test in predicting PCa aggressiveness $236[11,27,28]$ has had a significant impact on the diagnostic pathway of this disease [2].

237 When considering the field of PCa treatment and oncological outcomes, several 238 efforts are being made to provide evidence supporting the use of biomarkers to improve 239 patients' risk stratification and treatment choice [29,30]. Nonetheless, despite the strong 240 role of mpMRI as an independent predictor of clinically significant $\mathrm{PCa}$ (csPCa) 241 [11,31,32], data supporting the use of mpMRI endotypes, categorizing mpMRI severity 242 based on suspicion scale, as a prognostic factor of oncological outcomes after primary 243 treatment is still scarce.

244 A recently published meta-analysis assessing the role of mpMRI in predicting 245 oncological outcomes after EBRT showed that extracapsular extension, seminal vesicle 246 invasion, larger TV, and involvement of the prostate apex were significantly associated 247 with BCR rate [6]. Furthermore, the authors showed that lymph-node invasion at mpMRI 248 was significantly associated with prostate cancer specific-mortality (PCSM). Nonetheless, 249 this study was limited by some issues that deserve discussion: 1) the authors were not able 
250 to test some of the mpMRI factors that are currently considered the main predictors of PCa

251 aggressiveness (e.g. PI-RADS score) but differently the study assessed the prognostic value

252 of mpMRI staging parameters rather than qualitative characteristics of the mpMRI

253 abnormality; 2) pooled analyses of HRs were performed including non-adjusted analyses

254 that might have introduced significant bias leading to unreliable results; 3) only studies

255 relying on EBRT cohorts were included.

256 Given the high heterogeneity of studies in this field, we aimed to identify qualitative

257 or quantitative mpMRI factors that could represent independent predictors of oncological

258 outcomes to support their inclusion in the risk assessment of patients referred for treatment

259 for PCa.

260 Based on our findings, several observations can be made. First, the standardized PI-

261 RADS scoring system, represented an independent predictor of BCR after RP in the

262 majority of the included studies particularly when PI-RADS score was tested as a

263 continuous variable, even when adjusting for disease aggressiveness. Of note, despite all

264 the studies relied on PI-RADS score, residual heterogeneity, mainly due to differences in

265 cut-offs of PI-RADS category stratification, prevented the calculation of a pooled HR for

266 mpMRI scoring system. Nonetheless, we showed that a higher PI-RADS score was

267 associated with increased risk of BCR after RP, particularly when PI-RADS 5 and PI-

268 RADS 3 lesions were compared to lesions with lower PI-RADS score and with negative 269 mpMRI, respectively [19,24].

270 The clinical implication of these findings are twofold: 1) the inclusion of PI-RADS

271 score in post-operative outcomes predictive tools should be reinforced. This will likely

272 allow to more accurately identify patients with a higher risk of unfavorable outcomes after 
273 treatment in order to better counsel patients willing to receive treatment and draw patient-

274 tailored therapeutic decisions; 2) as suggested by previous studies [33-35], PCa lesions'

275 features at mpMRI, and more specifically their grade of suspicion defined according to

276 standardized reporting systems [9] might be related to aggressiveness at a sub-cellular level

277 (e.g. genomic, metabolic). However, due to the high between-studies heterogeneity which

278 did not allow to perform a pooled meta-analysis of the mpMRI grading score on post-

279 operative outcomes, an overall figure of the cumulative effect of mpMRI PI-RADS score 280 on BCR could not be assessed.

281 Second, we failed to demonstrate a statistically significant relationship between 282 mpMRI TV and BCR after RP for PCa (HR: 0.99; $\mathrm{p}=0.84$ ). Nowadays, providing reliable 283 results regarding the role of mpMRI TV as an oncological prognostic factor is key, 284 particularly given the growing interest and evidence on tissue-preserving therapies like 285 focal therapy for PCa and its promising mid-term results [36]. Historically, PCa TV, 286 measured at whole-mount pathology, has been always considered both a predictor of $\mathrm{PCa}$ 287 aggressiveness [37] and disease recurrence as also demonstrated in a meta-analysis carried 288 out by Meng et al. [38]. When considering PCa TV measured at mpMRI, results are 289 controversial. Recently, Woo et al. [6] showed that mpMRI TV was significantly correlated 290 with BCR after EBRT. However, as aforementioned, Woo et al. provided pooled-analyses 291 that suffered by some limitations [15]. Our findings might be explained by the known 292 issues with the reliability of PCa TV when measured at mpMRI. First, most of the studies 293 assessing the ability of mpMRI in estimating tumor volume measured at whole-mount 294 pathology showed a significant underestimation, particularly for small lesions [39-42].

295 Second, the mpMRI sequence that best estimates tumor volume still needs to be defined, 
296 with the most reliable method appearing to be to measure PCa TV in the sequence where 297 the index lesion is best visible [43,44]. Lastly, even though mpMRI reaches good accuracy 298 in detecting PCa index lesions, the per-lesion sensitivity, namely the ability to identify 299 smaller PCa foci, is moderate at best [45,46]. In light of our findings, the use of PCa TV 300 measured at mpMRI needs to be further investigated and standardized in order to become 301 a useful and reliable predictive tool for oncological outcomes after treatment.

302 Finally, when attempting to explore outcomes with a more significant clinical 303 impact as disease progression or cancer-specific mortality, few or no reliable studies were 304 found. Therefore, no conclusions can be driven by these results.

305 Despite its strengths, mainly due to the strict selection criteria for the studies 306 included, this study is not devoid of limitations. First, due to the retrospective nature and 307 the wide range of study periods, against the relatively recent introduction of mpMRI for 308 diagnostic purposes, the heterogeneity among studies is significant [47]. Also, most of the 309 studies did not provide any information regarding mpMRI-targeted biopsy. Second, given 310 that the majority of studies assessed cohorts of men receiving RP, pooled analyses on the 311 role of mpMRI in men receiving other treatments were not possible. For this specific reason 312 our findings should be carefully considered when different primary treatments are taken 313 into account. Third, the median follow-up of the studies included was probably too short 314 to provide meaningful oncological outcomes in the field of PCa (range: 25 - 109 months).

315 Furthermore, the numbers of men experiencing the outcomes tested were quite low to 316 provide highly reliable adjusted analyses (Table 1). Fourth, given the multi-center nature 317 of this study, it was not possible to take into account the inter-reader agreement of mpMRI 318 reporting among different centers. Finally, few or no studies assessing mpMRI as a 
319 predictor of either disease progression or PCSM met the inclusion criteria for the purpose

320 of a meta-analysis. This limitation prevented us from drawing any reliable conclusion for

321 these specific topics.

322

\section{$323 \quad$ 5. Conclusions}

324 Among the clinical predictors of oncological outcomes after RP for PCa, PI-RADS

325 score seems to represent a promising independent prognostic factor. More specifically, the

326 PI-RADS score is significantly correlated with the rate of BCR in most of the studies and

327 it should be taken into account to identify men at higher risk of unfavorable outcomes. On

328 the other hand, there is no evidence to support the role of PCa TV at mpMRI as a predictor

329 of oncological outcomes after RP for PCa. Widespread of standardized, high-quality

330 mpMRI protocols and further investigation in the emerging field of artificial intelligence

331 is mandatory [47]. Further studies are needed to clarify the role of mpMRI suspicion as a

332 potential contributing factor in predictive models. 


\section{Acknowledgments}

343 Study conception and design: Armando Stabile, Elio Mazzone, Giuseppe O Cirulli,

344 Francesco De Cobelli, Jeremy Grummet, Harriet C Thoeny, Mark Emberton, Morgan

345 Pokorny, Peter A Pinto, Samir S Taneja

346 Acquisition of data: Armando Stabile, Giuseppe O Cirulli, Elio Mazzone

347 Analysis and interpretation of data: Armando Stabile, Elio Mazzone, Giuseppe O

348 Cirulli, Francesco De Cobelli, Jeremy Grummet, Harriet C Thoeny, Mark Emberton,

349 Morgan Pokorny, Peter A Pinto, Samir S Taneja

350 Drafting of the manuscript: Armando Stabile

351 Critical revision of the manuscript for important intellectual content: Francesco De

352 Cobelli, Jeremy Grummet, Harriet C Thoeny, Mark Emberton, Morgan Pokorny, Peter A

353 Pinto, Samir S Taneja

354 Statistical analysis: Armando Stabile

355 Obtaining funding: None

356 Administrative, technical, or material support: None

357 Supervision: Samir S Taneja

358 Other: None

359

360 Financial disclosures: Harriet C Thoeny was supported by: Swiss National Science

361 Foundation, Number: 32003B_176229/1. Mark Emberton receives research support from

362 the United Kingdom's UCLH/UCL National Institute of Health Research (NIHR)

363 Biomedical Research Centre. He was awarded NIHR Senior Investigator status in 2013. 


\section{Figure legend}

366 Figure 1: Flow diagram showing the outcome of the intial searches resulting in the full

367 studies included in the review (from databases inception until September $1^{\circ} 2020$ )

$368 *$ A study can assess relationship between mpMRI scoring system and/or tumor and BCR

369 or other survival outcomes.

370 Figure 2: Overall summary of risk of bias assessment across studies based on QUIPS

371 criteria. QUIPS = Quality in Prognostic Studies tool

372 Figure 3: Forest plot for predictive value of mpMRI scoring system for biochemical

373 recurrence. $\mathrm{RP}=$ radical prostatectomy

374 Figure 4: Forest plot for predictive value of mpMRI tumor volume for biochemical

375 recurrence. $\mathrm{RP}=$ radical prostatectomy; $\mathrm{TS}=$ tumor size; $\mathrm{TV}=$ tumor volume; $\mathrm{HIFU}=$

376 High intensity focused ultrasound

377 Suppl Figure 1: Risk of bias assessment for each study according based on QUIPS criteria.

378 QUIPS = Quality in Prognostic Studies tool 


\section{References}

389 [1] Stabile A, Giganti F, Rosenkrantz AB, et al. Multiparametric MRI for prostate $390 \quad$ cancer diagnosis: current status and future directions. Nat Rev Urol 2019.

391 [2] European Urology Association. EAU Guidelines on Prostate Cancer 2020.

392 [3] Martini A, Gupta A, Lewis SC, et al. Development and Internal Validation of a 393 Side Specific, Multiparametric MRI-based Nomogram for the Prediction of $394 \quad$ Extracapsular Extension of Prostate Cancer. BJU Int 2018.

395 [4] Gandaglia G, Ploussard G, Valerio M, et al. A Novel Nomogram to Identify 396 Candidates for Extended Pelvic Lymph Node Dissection Among Patients with 397 Clinically Localized Prostate Cancer Diagnosed with Magnetic Resonance 398 Imaging-targeted and Systematic Biopsies. Eur Urol 2019;75:506-14.

399 [5] Campbell JM, Raymond E, O’Callaghan ME, et al. Optimum Tools for Predicting 400 Clinical Outcomes in Prostate Cancer Patients Undergoing Radical Prostatectomy: 401 A Systematic Review of Prognostic Accuracy and Validity. Clin Genitourin $402 \quad$ Cancer 2017;15:e827-34.

403 [6] Woo S, Han S, Kim T, et al. Prognostic Value of Pretreatment MRI in Patients 404 With Prostate Cancer Treated With Radiation Therapy: A Systematic Review and 405 Meta-Analysis. AJR Am J Roentgenol 2020;214:597-604.

406 [7] Barentsz JO, Richenberg J, Clements R, et al. ESUR prostate MR guidelines 2012. $407 \quad$ Eur Radiol 2012;22:746-57.

408 [8] Renard-Penna R, Mozer P, Cornud F, et al. Prostate Imaging Reporting and Data 409 System and Likert Scoring System: Multiparametric MR Imaging Validation 410 Study to Screen Patients for Initial Biopsy. Radiology 2015;275:458-68. 
411 [9] Barentsz JO, Weinreb JC, Verma S, et al. Synopsis of the PI-RADS v2 Guidelines

412 for Multiparametric Prostate Magnetic Resonance Imaging and Recommendations

$413 \quad$ for Use. Eur Urol 2015;69:41-9.

414 [10] Turkbey B, Rosenkrantz AB, Haider MA, et al. Prostate Imaging Reporting and

415 Data System Version 2.1: 2019 Update of Prostate Imaging Reporting and Data

$416 \quad$ System Version 2. Eur Urol 2019;0232:1-12.

417 [11] Kasivisvanathan V, Stabile A, Neves JB, et al. Magnetic Resonance Imaging-

418 targeted Biopsy Versus Systematic Biopsy in the Detection of Prostate Cancer: A

$419 \quad$ Systematic Review and Meta-analysis. Eur Urol 2019;44.

420 [12] Ahmed HU, El-Shater Bosaily A, Brown LC, et al. Diagnostic accuracy of multi-

421 parametric MRI and TRUS biopsy in prostate cancer (PROMIS): a paired

422 validating confirmatory study. Lancet 2017;389:815-22.

423 [13] Moher D, Shamseer L, Clarke M, et al. Preferred reporting items for systematic

424 review and meta-analysis protocols (PRISMA-P) 2015 statement. Syst Rev

$425 \quad 2015: 1-9$.

426 [14] Scher HI, Halabi S, Tannock I, et al. Design and End Points of Clinical Trials for

427 Patients With Progressive Prostate Cancer and Castrate Levels of Testosterone:

428 Recommendations of the Prostate Cancer Clinical Trials Working Group. J Clin

$429 \quad$ Oncol 2008;26:1148-59.

430 [15] Deeks J, Higgins J, Altman D. Analysing data and undertaking meta-analyses.

431 Cochrane Handb. Syst. Rev. Interv. version 6.0, 2019.

432 [16] DerSimonian R, Laird N. Meta-analysis in clinical trials. Control Clin Trials

$433 \quad 1986 ; 7: 177-88$. 
434 [17] Higgins P, Thompson S, Deeks J, Altman D. Measuring inconsistency in $435 \quad$ knowledgebases. BMJ 2003;27:159-84.

436 [18] Hayden J, Coté P, Bombardier C. Evaluation of the Quality of Prognosis Studies in 437 Systematic Reviews. Ann Intern Med 2006;144:427-38.

438 [19] Gandaglia G, Ploussard G, Valerio M, et al. Prognostic Implications of

439 Multiparametric Magnetic Resonance Imaging and Concomitant Systematic

440 Biopsy in Predicting Biochemical Recurrence After Radical Prostatectomy in

441 Prostate Cancer Patients Diagnosed with Magnetic Resonance Imaging-targeted

442 Biopsy. Eur Urol Oncol 2020.

443 [20] Zhang YD, Wu CJ, Bao ML, et al. MR-based prognostic nomogram for prostate $444 \quad$ cancer after radical prostatectomy. J Magn Reson Imaging 2017;45:586-96.

445 [21] Zhang Z. Survival analysis in the presence of competing risks. Ann Transl Med J

446 Cardiovasc Med Hemodial Int J Transl Med Crit Care Int J Clin Pract J Crit Care $447 \quad$ Ann Transl Med 2017;55.

448 [22] Park SY, Oh YT, Jung DC, et al. Prediction of biochemical recurrence after radical 449 prostatectomy with PI-RADS version 2 in prostate cancers: initial results. Eur $450 \quad$ Radiol 2016;26:2502-9.

451 [23] Hattori S, Kosaka T, Mizuno R, et al. Prognostic value of preoperative 452 multiparametric magnetic resonance imaging (MRI) for predicting biochemical 453 recurrence after radical prostatectomy. BJU Int 2014;113:741-7.

454 [24] Kim R, Kim CK, Park JJ, et al. Prognostic significance for long-term outcomes 455 following radical prostatectomy in men with prostate cancer: Evaluation with 456 prostate imaging reporting and data system version 2. Korean J Radiol 
458 [25] Fitzmaurice C, Allen C, Barber RM, et al. Global, Regional, and National Cancer Incidence, Mortality, Years of Life Lost, Years Lived With Disability, and

462 [26] Turchan WT, Kauffmann G, Patel P, et al. PI-RADS score is associated with

[27] van der Leest M, Cornel EB, Israel B, et al. Head-to-head Comparison of Transrectal Ultrasound-guided Prostate Biopsy Versus Multiparametric Prostate

[29] Cucchiara V, Cooperberg MR, Dall M, et al. Genomic Markers in Prostate Cancer

[28] Rouvière O, Puech P, Renard-penna R, et al. Use of prostate systematic and targeted biopsy on the basis of multiparametric MRI in biopsy-naive patients Decision Making. Eur Urol 2017;73:572-82. (MRI-FIRST): a prospective, multicentre, paired diagnostic study. Lancet Oncol RNA acceptance in prostate cancer initiation, progression, and its clinical utility in disease management. Eur Urol 2019;76:546-59. 
480 [31] Ahmed HU, Bosaily AE, Brown LC, et al. Diagnostic accuracy of multi-

481 parametric MRI and TRUS biopsy in prostate cancer ( PROMIS ): a paired

$482 \quad$ validating confi rmatory study. Lancet $2017 ; 6736: 1-8$.

483 [32] Kasivisvanathan V, Rannikko AS, Borghi M, et al. MRI-Targeted or Standard

484 Biopsy for Prostate-Cancer Diagnosis. N Engl J Med 2018;378:1767-77.

485 [33] Houlahan KE, Salmasi A, Sadun TY, et al. Molecular Hallmarks of

486 Multiparametric Magnetic Resonance Imaging Visibility in Prostate Cancer. Eur

$487 \quad$ Urol 2019;76:18-23.

488 [34] Parry MA, Srivastava A, Ali A, et al. Genomic Evaluation of Multiparametric

489 Magnetic Resonance Imaging-visible and -nonvisible Lesions in Clinically

$490 \quad$ Localised Prostate Cancer. Eur Urol Oncol 2018;2:1-11.

491 [35] Norris JM, Simpson BS, Parry MA, et al. Genetic landscape of prostate cancer

492 conspicuity on multiparametric MRI: a protocol for a systematic review and

493 bioinformatic analysis. BMJ Open 2020;10:e034611.

494 [36] Stabile A, Orczyk C, Hosking-jervis F, et al. Medium-term oncological outcomes

495 in a large cohort of men treated with either focal or hemi-ablation using high-

496 intensity focused ultrasonography for primary localized prostate cancer. BJU Int

4972019.

498 [37] Ahmed HU, Hu Y, Carter T, et al. Characterizing Clinically Significant Prostate

499 Cancer Using Template Prostate Mapping Biopsy. J Urol 2011;186:458-64.

500 [38] Meng Y, Li H, Xu P, Wang J. Do tumor volume, percent tumor volume predict

501 biochemical recurrence after radical prostatectomy? A meta-analysis. Int J Clin

$502 \quad$ Exp Med 2015;8:22319-27. 
503 [39] Le Nobin J, Orczyk C, Deng FM, et al. Prostate tumour volumes: Evaluation of the 504 agreement between magnetic resonance imaging and histology using novel co505 registration software. BJU Int 2014;114:E105-12.

506 [40] Nobin J Le, Rosenkrantz AB, Villers A, et al. Image Guided Focal Therapy for 507 Magnetic Resonance Imaging Visible Prostate Cancer: Defining a 3-Dimensional 508 Treatment Margin Based on Magnetic Resonance Imaging Histology Co$509 \quad$ Registration Analysis. J Urol 2015;194:364-70.

510 [41] Cornud F, Khoury G, Bouazza N, et al. Tumor target volume for focal therapy of 511 prostate cancer - Does multiparametric magnetic resonance imaging allow for a 512 reliable estimation? J Urol 2014;191:1272-9.

513 [42] Turkbey B, Mani H, Aras O, et al. Correlation of magnetic resonance imaging $514 \quad$ tumor volume with histopathology. J Urol 2012;188:1157-63.

515 [43] Sun C, Chatterjee A, Yousuf A, et al. Comparison of T2-Weighted Imaging, DWI, 516 and Dynamic Contrast-Enhanced MRI for Calculation of Prostate Cancer Index

517 Lesion Volume: Correlation With Whole-Mount Pathology. AJR Am J Roentgenol $518 \quad 2019 ; 212: 351-6$.

519 [44] Christie DRH, Sharpley CF. How accurately can multiparametric magnetic 520 resonance imaging the tumour volume of a prostate cancer? J Med Imaging Radiat $521 \quad$ Oncol 2020:1-10.

522 [45] Johnson DC, Raman SS, Mirak SA, et al. Detection of Individual Prostate Cancer 523 Foci via Multiparametric Magnetic Resonance Imaging. Eur Urol 2018;75:712-20.

524 [46] Stabile A, Dell'Oglio P, Cobelli F De, et al. Association Between Prostate Imaging 525 Reporting and Data System ( PI-RADS ) Score for the Index Lesion and 
526 Multifocal, Clinically Significant Prostate Cancer. Eur Urol Oncol 2018;1:1-8.

527 [47] Stabile A, Giganti F, Kasivisvanathan V, et al. Factors Influencing Variability in 528 the Performance of Multiparametric Magnetic Resonance Imaging in Detecting

529 Clinically Significant Prostate Cancer: A Systematic Literature Review. Eur Urol $530 \quad$ Oncol 2020;3. 